\title{
TOWARDS A Reliable EleCtion Algorithm For VANETS NETWORKS
}

\author{
OuafaMahma $^{1}$ and Ahmed Korichi ${ }^{2}$ \\ ${ }^{1 \& 2}$ Department of Computer Science and Information Technology, KasdiMerbah \\ University, Ouargla, Algeria
}

\begin{abstract}
The alert data dissemination is one of principal's several works in VANETs network research. This latter is composed of autonomous entities characterized by high speed and predictable regular mobility "intelligent vehicles".

Indeed, several studies are proposed for improvement security in this networks. Which is considered one of the most important objective of Intelligent Transport Systems (ITS), that are using different technologies and ways to meet this goal, based mainly on to the information disseminated between network's vehicles related studies.

In this context, The goal in this paper is modelling and simulate an election algorithm for VANET networks, to improve the warning data dissemination in order to achieve a passenger security service on the routes. Where we choose the modelling and simulation approach to justified our technique.
\end{abstract}

\section{KEYWORDS}

VANET; Election algorithm ; data dissemination; simulation; modelling.

\section{INTRODUCTION}

Intelligent Transportation Systems (ITS) denote the applications of new information technology and communications in the transport field [1].

One of the objectives of these systems: passenger safety assurance on the roads, this point forms the principle of our contribution .where we provide an algorithm to facilitate the safety information dissemination among vehicles travelling in a geographic area.

So, the need to select a vehicle or more among several to act as a transmitter (s) for each alert message rebroadcast session among network's vehicles. That guided authors to choose a solution compatible with this type of systems.

Therefore, we chose the elections algorithms in distributed systems as a solution to solve this problem. These algorithms are one of the essential paradigms used in distributed systems (mutual exclusion, election ...).

In this paper, we start with an introduction to election algorithm in distributed systems, few algorithms, defining our approach, and finally results. 


\section{Distributed AlgorithM "Distributed Election"}

Distributed algorithms are a paradigm be applied to a set of computers, or sites, process or generally collection of autonomous entities. They are interconnected, can communicate through the exchange of messages through a communication system. The specific composition of these sites (local memory and other components) allows them to be autonomous and able to communicate, in this system there is no centralized management or coordination! (No concept of a global clock or a shared memory) $[3,2]$.

Indeed, the lack of synchronization affects the incompatibility intern of components and the effectiveness and reliability of this system, since all the processes need to interact with an coordinator.[4]

One of the major problems in this type of systems is the management of the collection. In other words some tasks and activities in the collection require the selection of one or more processes to play a unique and important role (coordination, control, management ...) in the group to also ensure the proper functioning of this totality.

Therefore, multiple solutions and algorithms are mentioned example of mutual exclusion algorithms and algorithms election.

Several definitions are available but are generally might know that a distributed system is the collection of process exchanging messages by a communication system.

Distributed algorithms are different; we chose it in these papers to select one of nods to able as coordinator. In order to achieve a new algorithm for managing, the warning information dissemination in VANETs networks.

\section{The Purpose of Choosing This Paradigm}

The election algorithm is an important paradigm of distributed systems, which meant the selection, choice, election (election) process or entity or more among others for care or charge for a specific function or role in the whole.

Our system (VANET) is a combination of vehicles (autonomous entities) communicates each to other through messages .Our specification is about the warning messages distributed in VANET network. It is a distributed system where the components are intelligent and even moving, moved at a different and very high speed. It is decentralized where no centralized control is.

In addition to the above and generally, our purposes in this paper are the same purpose of used the election method: needed to require one act as coordinator or sequencer, someone for a special role in the collection, needed to a common topology picture of overall system.

On the other part, the need to use a vehicle in a network among others to act as emitter / initiator (leader) at every turn or session broadcast / rebroadcast the alert message between this vehicles, Has made us choose an optimal solution to this situation. Therefore, we chose the algorithms election in distributed systems that form an essential paradigm in this type of systems.

\section{Assumptions of Election Paradigm}

- When the the coordinator crashes or retires in the system, Here it became necessary need to adopt an election.

- Any node can serve as coordinator. 
- Any node can initiate the election.[5,6]

- The numbered uniquely of processes: each process in collection has an unique identification (example of network address). If all processes have exactly the same number, with no distinguishing characteristics, so there is no need and no reason in basis to select one to be special.

- Basically this technique works on the selection on the basis of the biggest defined as manager of the group.

- All processes know about each other: every process knows the process number of every other process. $[3,5,6,7,8]$

\section{Distributed Election Existing Algorithms}

Generally there are several election algorithms, among them:

\section{Bully Algorithm}

This algorithm is [9, 10,5,6]proposed in 1982 by Hector Garcia Molina .Generally, it is assumed that every process knows the priority number of every other process in the system, and The communication subsystem does not fail.

Example:

In the figure 1 . At this system, node ' 6 ' is the coordinator, because it is the highest numbered node. But In the figure two, ' 6 ' leaves, so we need a coordinator?

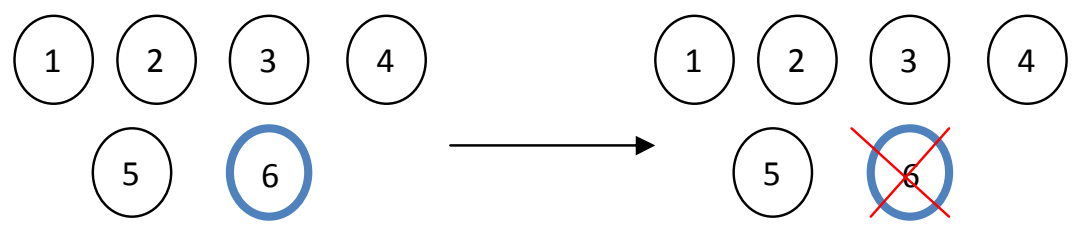

Figure 1

The node ' 3 ' decides it wants to be the coordinator: it sends an election message to all higher numbered processes.

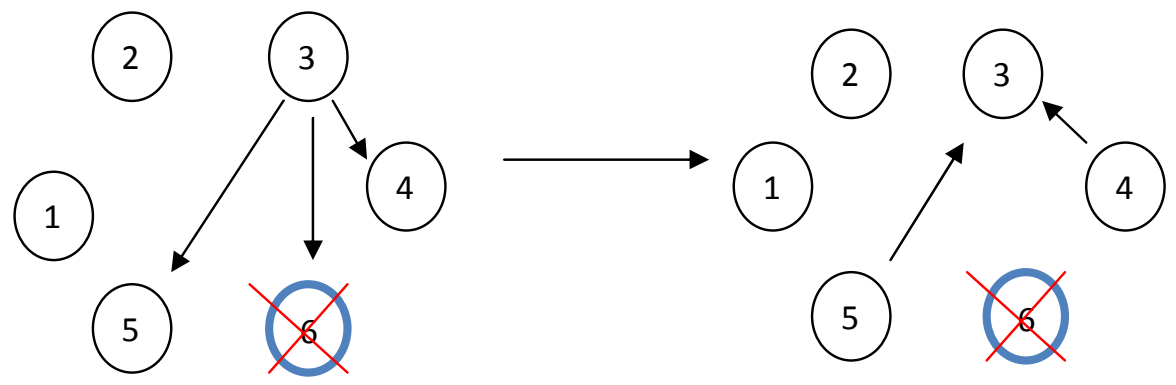

Figure 2

If no response, 3 takes over as coordinator, but here! Nodes ' 4 ', and ' 5 ' are stillyet, so they reply node ' 3 ' that "I am here". ( see figure 2) 
International Journal of Computer Science \& Information Technology (IJCSIT) Vol 8, No 2, April 2016

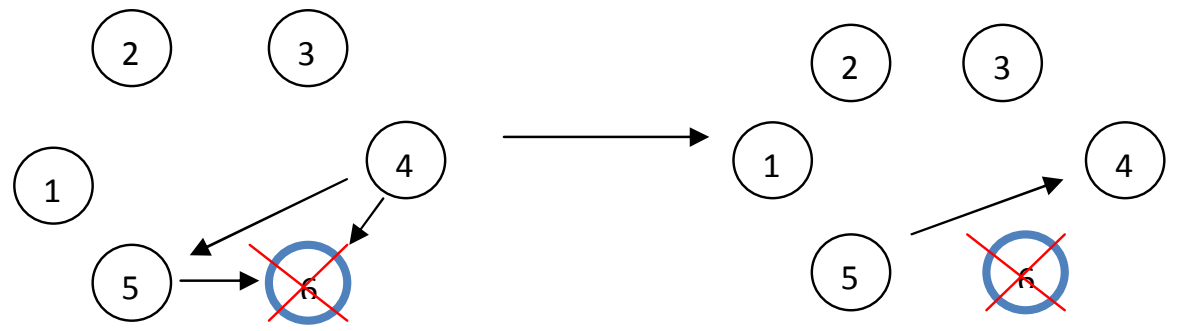

Figure 3

In figure 3 , the nodes ' 4 ' and ' 5 ' sends election messages to all higher numbered processes. In this case, node ' 4 ' sends an election to node ' 5 ' and ' 6 '. And ' 5 ' just sends an election to node ' 6 '. But 6 is not active .Now, since node ' 5 ' has the higher number and it is still, then node ' 5 ' tells node ' 4 ' that it is still here (figure 3). And the node ' 6 ' has not replied to node ' 5 '

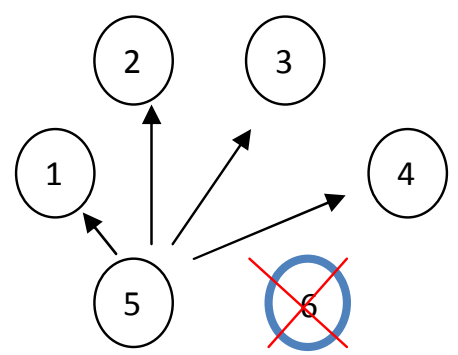

Figure 4

Finally, this node ' 6 ' is the new coordinator. It is forced to tell all the other nodes.

\section{Ring Algorithm}

This algorithm is based on the ring election algorithms presented in Tanenbaum1995, Silberschatz and Galvin 1994[ 9, 10, 5, 6, 7, 8 ].Assuming that all system processes are organized in a logical ring. All messages related to the election algorithm are always passed only in one direction (Ring unidirectional) .All process in the system knows the structure of the ring. So that while trying to circulate message over the ring (in case of the sender successor process is down) .the sender can skip over the successor the one after that, until an active member is located: We have the following system,

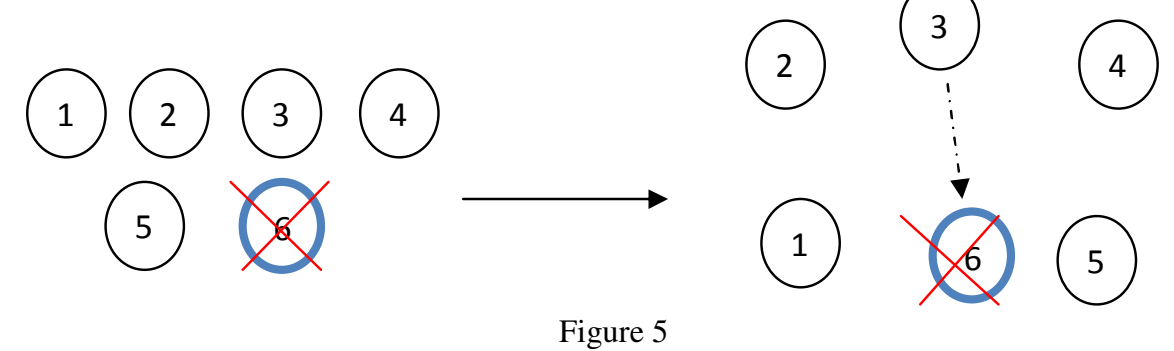

1- When a process 3 sends request message to coordinator $\left(n^{\circ} 6\right)$, this last no reply in fixed timeout period. It assumes that the coordinator has crashed. 

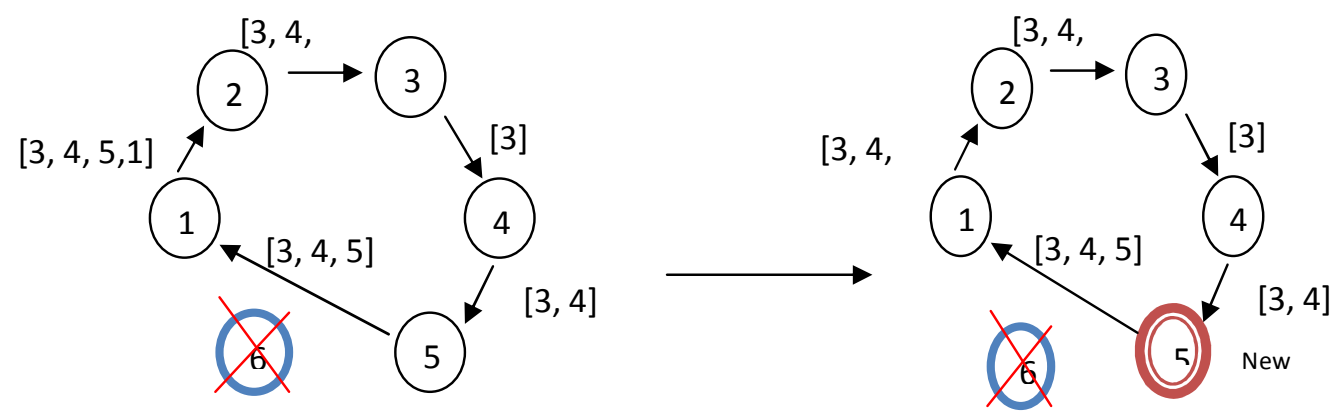

Figure 6

2- Nod 3 initializes an election to its successor (currently actives) message contains the priority number (3) and so on (see figure 6) .

3 - When process 3 receives its own election message, the message contains the list of all processes actives currently

4- Therefore of the processes in this list, it elects the process having the highest priority number as the new coordinator.( see figure 6)

We cannot rely on the effectiveness of this technique in the case of a VANET network, as well as we can have difficulty access to the information quickly and timely. This because of the characteristics of this type of network "VANET" Compared to wired networks as the network topology constantly changing, the vast network, lack of communication infrastructure, unstable network nodes and their great speed and their distribution caused the difficulty to keep track of the others continuously by any vehicle.so this algorithms aren't suitable for our case.

\section{- Wireless Election Algorithms ( in Wireless And Very Large-Scale Systems)}

Our system focuses on direct communication between components also depends on short-range wireless communication between vehicles, and are constructed on-the-fly dedicated to this type of network. Also communication and information technology in this system mainly depends on the choice of one car out of $\mathrm{N}$ vehicles, so that there will be conditions achieved them. Its enables the car charged with the choosing a car for each tour of the dissemination of the information. $[9,10$, $5,6]$

We will use the same principle used in large ad hoc network algorithms with some changes for correspond to our system, which based on:

- The election process (an ELECTION message sending out ) begins with any node in the group.

- The node receives the ELECTION message for the first time; it forwards to neighbours, and designates the sender as its parent and waits their responses. There lasts may carry source information.

- For any node, the second time receive election message, it just OKs it.

- At each stage the "most eligible" or "best" node will be passed along from receivers to source node.

- Upon the arrival of all the replies to the source, it is in a position to choose the new coordinator. After the selection process finishes (broadcast to all network nodes).

- In the event of a large number of election called (multiple source nodes), a node should participate in only one... $[3,9,10,5,6,7,8]$ 


\section{Dissemination Proposed Algorithm}

In this paper, we choose this last paradigm with simple changes in the methodology:

- Here the system goal is dissemination of security and warning information, we chose to divide the selection process on phases for short times to reduce the procedure, rather than make a choice in the big time.

- We relied in election process on one node charged the election and broadcasting of warning information at the same time. the election will be after obtain information about neighbors in range transmission of sender.

- Election is the result of several causes related to the distance between ends transmitter and the direction, regardless the speed, the reasons of the vehicle "chosen or sender" is parked for some reason, this will not affect because of the election process is cyclic, is not for a long-term and is for simple time.

- This system used once message of security distributed, with once sender for each turn.

\section{A. Assumptions}

- Each process i (vehicle), it has a variable "State i" which takes one of two following values: active, inactive (Participant or elected).

- Each process $\mathrm{i}$ (vehicle) has an identifier "ID i" unique, that defined at the beginning

- The time of communication finishes and unpredictable.

- No process failure.

- The communication will be through messages.

- The messages are not duplicated.

- Any vehicle can receive and sent messages.

- Processes running the same algorithm.

- The execution is begun and finished by only one process at a time; Unlike some election distributed algorithms that begins with a number one process (1..n).

- Each session of election, a new process will be selected (elected) in the end of this session, and finished with the sending a warning message by all direct neighbors in its transmission range.

- At each session, one message is broadcast at a time.

\section{B. Principle of the Algorithm}

- Each vehicle has information about these direct neighbors because of periodic hello messages broadcasted in the system. With temporary updates in list of neighbor.

- The transmitter checks its neighbor list, and checks which one satisfied the election conditions before the security message dissemination.

- After the election, the sender broadcasts security message with identification of vehicle elected for him informed of his role.

- When a vehicle receives the message and his identification does not equal to identification of vehicle elected then it will be active.

- If satisfies then this vehicle will be the next (new) diffuser.

\section{The Steps of the Algorithm}

I. Information: broadcast messages neighborhood

II. Election: the choice and selection of next transmitter 
III. Emission: security message broadcast by the transmitter.

IV. Exit: end of the session and out of his transmitter.

\section{The Process States in This System}

1. Process not receives a warning information message: status is inactive.

2. Process receives the warning message and not satisfied the conditions for election: state is active or participant.

3. Process informed message and satisfied the conditions for election: state is elected as the new transmitter.[11]

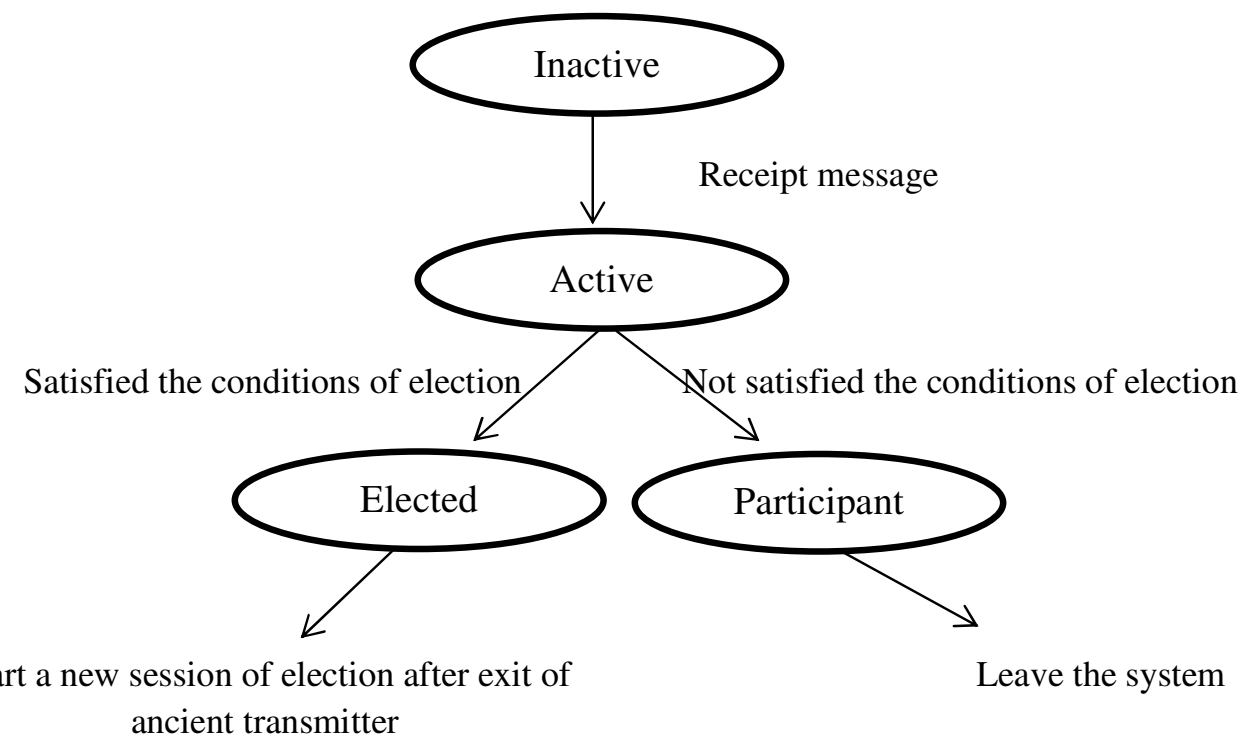

Figure 7. Process states

\section{E. The Results and Benefits of this Algorithm}

- The alert message received by a vehicle ensures inform this latter.

- (Continue to implement) the execution of this algorithm by the process with the use of notions of "distributed election" ensures any time the existence of a transmitter or distributor message.

- The division of election process on turns helps to keep the security message in the effect area and ensure information of all vehicles in the vicinity (the main goal).

- Election time sessions usually is useful, compatible with the main goal record and beneficial.

- Since the technique is based on sending a single message only for once phase, this will make the dissemination time record and small. Transmission and the choice will be in the same message, and by the same car makes them goes without in waiting time.

\section{Performance evaluation}

In this part we try to study the performance of proposed algorithm set by the system (GODD) in [11]. We use our proposed algorithm. Then analyzing and comparing the results found. 
We chose to use in this work the analytical study based on simulation results. The parameters measured are the number and percentage of informed vehicles before the risk area, and the rate of message disseminate in the system.

So our model is defined in the next section with simulation results .where we use the simulator OMNET ++ (Version: 4.3.1) and the simulator of traffic SUMO (0.17.1) and VEINS (2.2) to achieve our results.

\section{A. Simulation}

In this party, as we say in our precedent publication [11], we prefer to use OMNET ++ open source simulation library that is written in $\mathrm{C}++$. Which simple to use and it easy to learn.

\section{A.1 The Simulation Parameters}

To achieve the simulation scenarios, we chose the following parameters:

Table 1.Simulation Parameters.

\begin{tabular}{|l|l|}
\hline \multicolumn{1}{|c|}{ Description } & \multicolumn{1}{c|}{ value } \\
\hline Transmission range(R) & $250 \mathrm{~m}$ \\
\hline Straightroad & $7 \mathrm{~km}$ \\
\hline Mac layer & IEEE802.11 \\
\hline Safety distance & $250 \mathrm{~m}$ \\
\hline Effect distance & $10 \mathrm{Km}$ \\
\hline simulation time & $3000 \mathrm{~s}$ \\
\hline Start time of the warning event & $250 \mathrm{~s}$ \\
\hline
\end{tabular}

We omit the start time of the warning event to let the system reach a stable state considering the distribution of vehicles over the road. For both scenarios, when the warning event occurs, the beginner of dissemination is at the location of the safety line. [11]

\section{B. Results and Discussion}

Red Colored data is our model data but colored in bleu is the data of Ocast model in [12].

In figures10 and 11: different traffic value (measured density 1 to 15) vehicles are informed to $100 \%$ before the risk area (in both systems).

In this case the simulation results are (in Figures 8 and 9): Overall objective in this our study is the is to measure the flexibility, efficacy and usefulness of this algorithm and what it can add to the warning system. In this simulation, we see that the number of broadcasts and distributed data rate was very good.With Increased density, we note the algorithm flexibility and compatibility with a number of cars no matter how is large. 
International Journal of Computer Science \& Information Technology (IJCSIT) Vol 8, No 2, April 2016

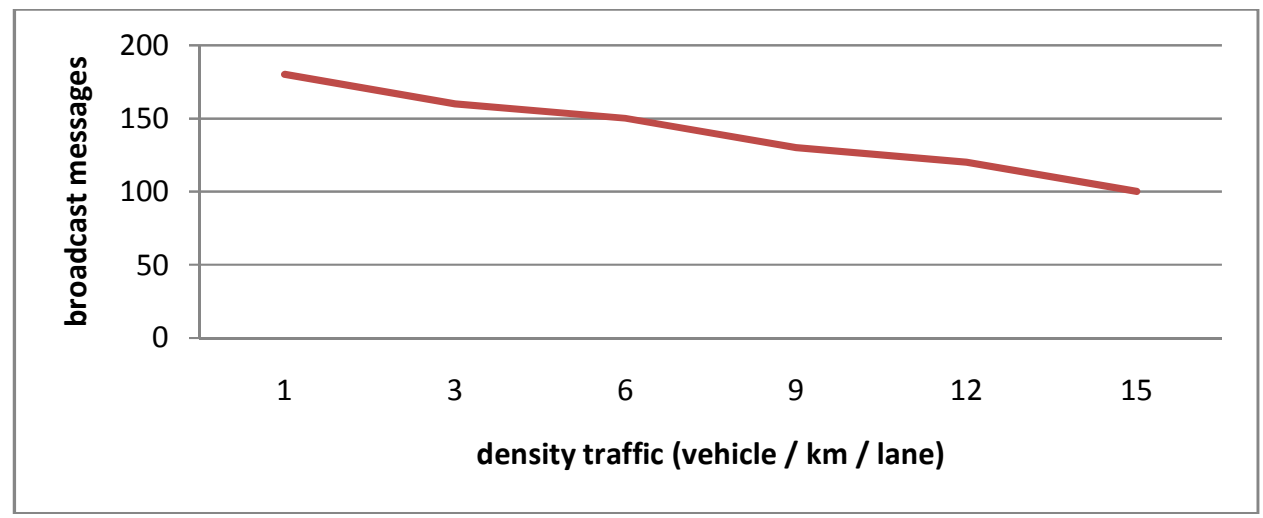

Figure 8.Broadcast vs. density

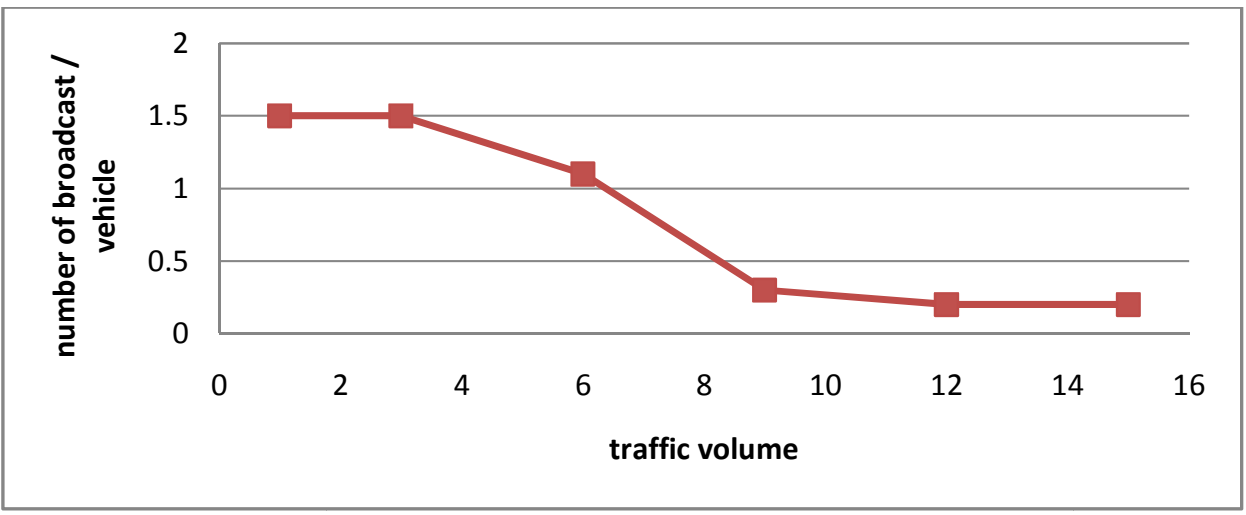

Figure 9. Broadcast/vehicle vs. traffic volume

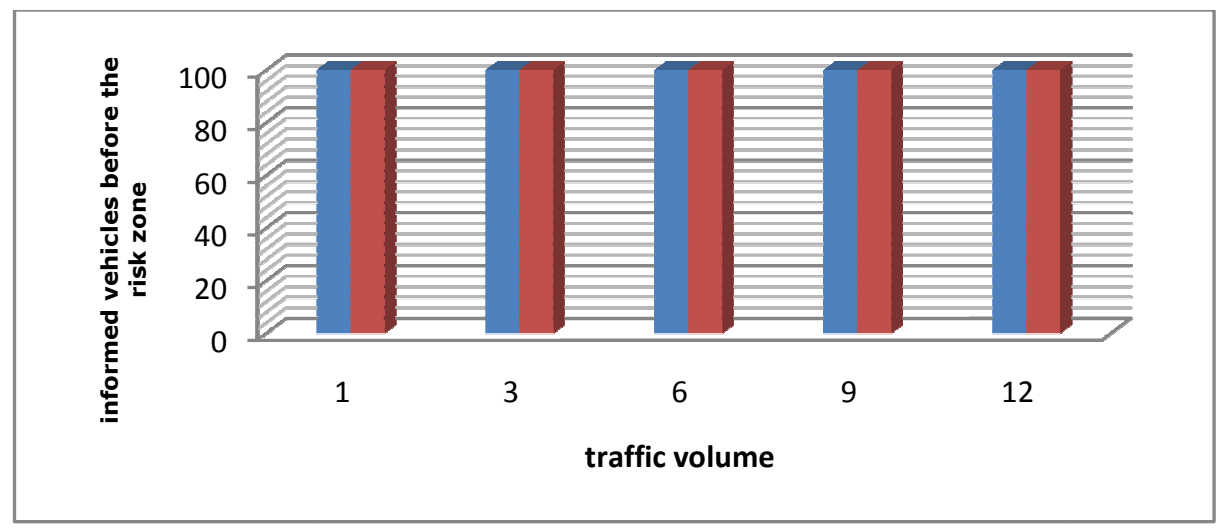

Figure.10 Informed vehicles vs. traffic volume 
International Journal of Computer Science \& Information Technology (IJCSIT) Vol 8, No 2, April 2016

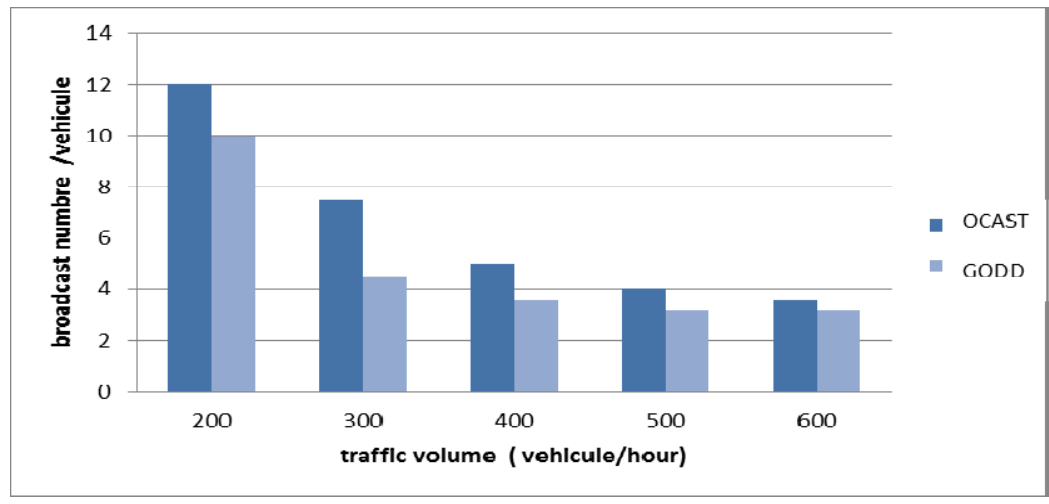

Figure .11Rates of broadcasts before they risk area[11].

Generally, in this communication system Geocast inter vehicles is interested in the warning information released as soon as possible quickly the source node to several recipients in a region geographically define to secure these vehicles [11].

We use strategies to achieve our objective, to inform all these points while reducing unnecessary broadcasts and ensure a favorable reception time.

\section{Conclusions}

We tried to present in this article an algorithm for election a leader in VANET networks used to improve the security data dissemination. Which is introducing a new contribution for more secure passengers in the roads.

\section{REFERENCES}

[1] V.Veronique .J. Colette(2012), «Hiérarchisation dans les réseaux ad hoc de véhicules », these.

[2] P.K .Sinha (2008), " Distributed Operating Systems Concepts and Design", Prentice-Hall of India private Limited.

[3] H.Katwala, S.Shah( 2012) ."Study on Election Algorithm in Distributed System", IOSR Journal of Computer Engineering (IOSRJCE), ISSN: 2278-0661, ISBN: 2278-8727Volume 7, Issue 6 (Nov. Dec. 2012), PP 34-39.2012.

[4] P BeaulahSoundarabai,JThriveni, K R Venugopal, L M Patnaik( 2013) . An improved leader election algorithm for distributed systems, International Journal of Next-Generation Networks (IJNGN) Vol.5, No.1.

[5] Pooja B. Raval1, Sanjay M. Shah (2012) " "Study of various Election algorithms on the basis of messagepassingapproach”.IOSR Journal of Computer Engineering (IOSRJCE), ISSN: 2278-0661, ISBN: 2278-8727Volume 8, Issue 1, PP 23-27.

[6] SachiChoudhary, DipeshSharma( 2012) A Comparative Analysis in Terms of Message Passing \& Complexity of Different Coordinator Selection Algorithms in Distributed System . International Journal of Advanced Research in Computer Engineering \& Technology (IJARCET), Volume 1, Issue 7.

[7] https://www.coursehero.com/file/6633143/A13Elections( 10/03/2016)

[8] http://fr.slideshare.net/sandpoonia/8-mutual-exclusion (10/03/2016)

[9] A.S Tanenbaum( 2007) . "Distributed Operating Systems: CONCEPTS AND DESIGN", Pearson Education.

[10] V.P. Gajre (2013) ."Comparison Of Bully election Algorithms In Distributed System”. International Journal of Scientific and Research Publications, Volume 3, Issue 9, September 2013 ,ISSN 22503153 
International Journal of Computer Science \& Information Technology (IJCSIT) Vol 8, No 2, April 2016

[11] O. Mahma , A. Korichi (2016), "Towards A New Approach Of Data Dissemination In Vanets Networks", The AIRCC's International Journal of Computer Science and Information Technology , ISSN: 0975 - 3826, Volume : 6 number : 2

[12] A. Benaidja ,S. Moussaoui and F. Naït-Abdesselam (2013) “An Optimal Broadcast of Warning Messages in Vehicular Ad Hoc Networks “, International Journal of Computer and Information Technology , ISSN: 2279 - 0764, Volume 02- Issue 05.

[13] Q. Yu and G. Heijenk .2008. "Abiding Geocast for Warning Message Dissemination in Vehicular Ad Hoc Networks", IEEE International Conference on Communications Workshops (ICC) Beijing, China, pp. 400-404.

[14] Min-Te Sun al.(2000) GPS-Based Message Broadcast for Adaptive Inter-vehicle communications, VTC Fall.

[15] S. Panichpapiboon, and W. Pattara-atikom (2011)“A Review of Information Dissemination Protocols for Vehicular Ad Hoc Networks", IEEE.

[16] http://documents.mx/documents/ad-hoc-mobile-wireless-networks.html(10/03/2016) 